\title{
The Use of ARM Data to Test An Improved Parameterization of Upper Tropospheric Clouds for Use in Climate Models
}

DOE/EP/61363--T1

DE92 014353

Progress Report

for Period December 1, 1991 through November 30, 1992

David A. Randall

Principal Investigator

Colorado State University

Department of Atmospheric Science

Fort Collins, CO 80523

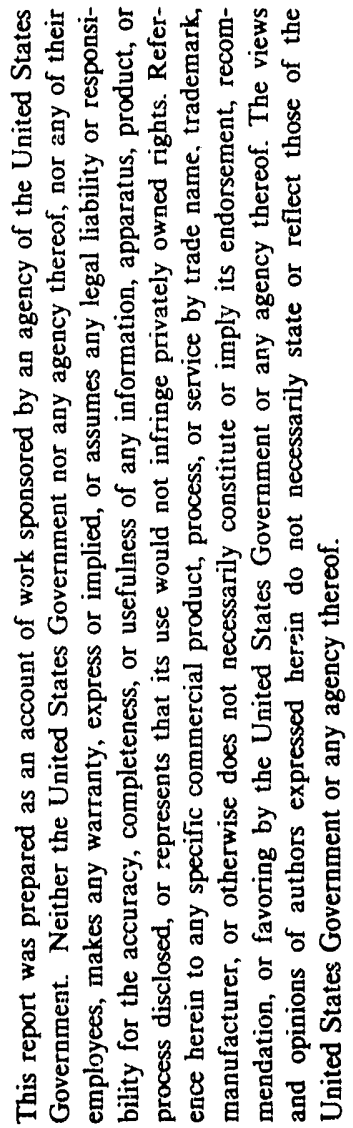

May 1992

Prepared for

THE U.S. DEPARTMENT OF ENERGY

AGREEMENT NO. DE-FG02-92ER61363 


\section{Introduction}

We have proposed to provide and further develop an advanced finite-difference climate model for use in CHAMMP. The model includes advanced parameterizations of cumulus convection, boundary-layer processes, cloud formation, and land-surface vegetation, as well as parameterizations of radiative transfer and gravity wave drag. Postprocessing codes and a user's guide will also be provided. This research is being conducted in collaboration with Professors C. R. Mechoso and A. Arakawa at the University of California at Los Angeles (UCLA).

The following research tasks are being carried out in support of CHAMMP:

a. Provide to CHAMMP a base-line finite-difference model and postprocessing codes for further development by the CHAMMP Science Team. A model has been provided to a research team working at DOE's Lawrence Livermore National Laboratory. Technical guidance has been provided to assist in optimization and further documentation of the code.

b. Provide to CHAMMP improved model physics to be developed in the course of our research project. Significant progress has already been made in development of improved parameterizations of cumulus convection (Randall and Pan, 1992) and the boundary layer (Randall, Shao, and Moeng, 1992), as well as land surface processes and prognostic cloud water. Some results were presented at the first CHAMMP Science Team Meeting. Some results obtained with the new cumulus parameterization are shown in Fig. 1. In these plots, the solid line (labeled "control," shows the results obtained with the earlier cumulus parameterization, while the other curves show how the results obtained with the new parameterization vary as a parameter called " $a$ " is changed. The results from the new model are asymptotically independent of a as a goes to zero. This is expected from the theory. Then new parameterization gives a distribution of precipitation that is more tightly organized, and the frequency of convective activity ("cumulus incidence") is much higher and apparently more realistic. Fig. 2 is a flow chart outlining the prognostic cloud water parameterization currently being tested in the GCM. As this diagram shows, the parameterization includes not only cloud water but also cloud ice, rain, and snow, all as prognostic variables.

c. Provide to CHAMMP improved computational methods for use in the model. We are currently experimenting with a geodesic grid on the sphere, in the context of a shallow water model. As illustrated in Fig. 3, the grid is symmetrical about the equator, thus preserving the symmetry of the governing equations. Multi-grid methods are being used to solve the vonicity and divergence equations efficiently.

d. Investigate the performance of current and to-be-developed physical parameterizations and computational methods at very high resolution. Xu (1991) has recently shown that the Arakawa-Schubert convective parameterization performs better at higher horizontal resolution. Further investigations of this type are being conducted, based in part on the use of UCLA's Cumulus Ensemble Model, which is now in use at both UCLA and CSU.

\section{Plans for 1993}

a. Provide to CHAMMP a base-line finite-difference model and postprocessing codes for further development by the CHAMMP Science Team. Additional code will be provided to DOE as warranted by progress in the model development efforts. 

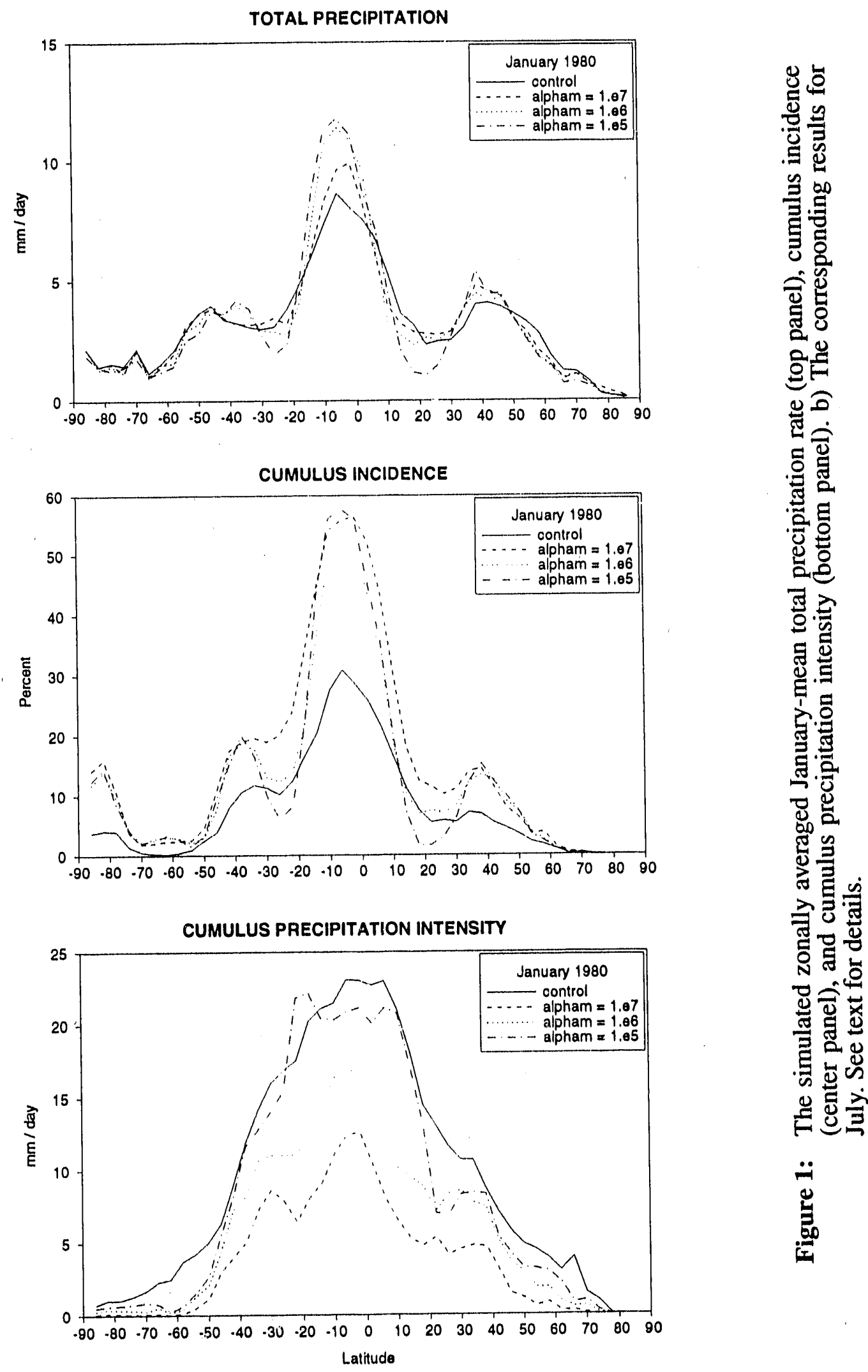


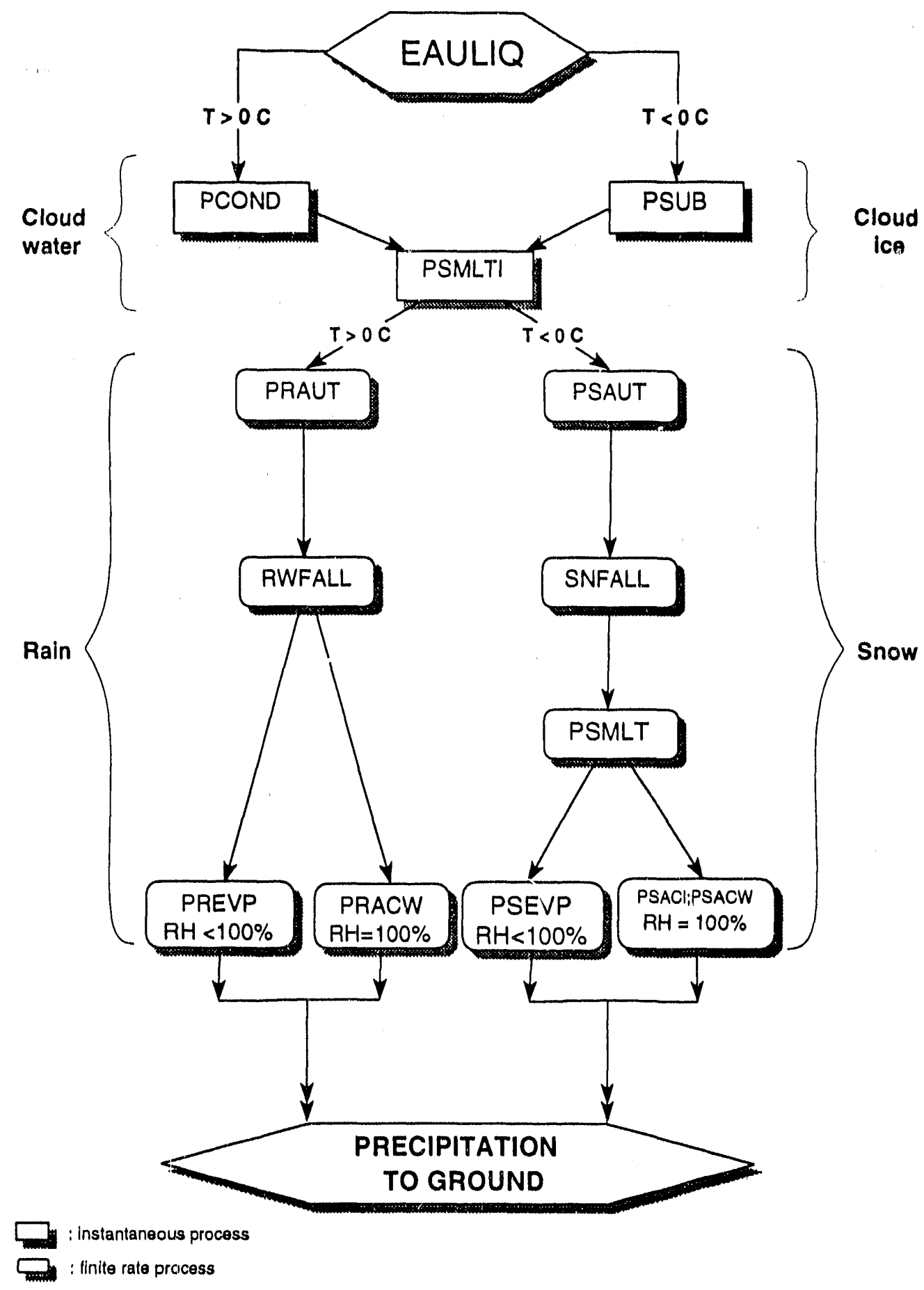

Figure 2: Flow chart summarizing the prognostic cloud water parameterization under development for the CSU GCM. 
b. Provide to CHAMMP improved model physics to be developed din the course of our research project. We will continue development and testing of our improved parameterizations of cumulus convection, the boundary layer, land surface processes, and prognostic cloud water. It is possible that at least one of these parameterizations can be tested by DOE's research team during 1993.

c. Provide to CHAMMP improved computational methods for use in the model. Although our geodesic-grid shallow model is currently running on a workstation and a Cray, we plan to port it to the Connection Machine at Los Alamos Naiional Laboratory. The code has been developed with such a port in mind from the beginning. This can be accomplished during 1993. Results from the model will be compared with results from a conventional finite-difference model and a spectral model. A Master's thesis based on this work will be completed no later than 1993.

d. Investigate the performance of current and to-be-developed physical parameterizations and computational methods at very high resolution. We plan to continue our resolution studies using the Cumulus Ensemble Model.

\section{REFERENCES AND BIBLIOGRAPHY}

Xu, K.-M., and D. A. Randall, 1992: The Semi-Empirical Basis of a Prognostic Cloud Parameterization for Use in Climate Models. Paper presented at the 11th International Conference on Clouds and Precipitation, Montreal, Canada.

Smith, L. D., and D. A. Randall, 1992: Inclusion of Cloud Microphysical Processes in the CSU General Circulation Model. Paper presented at the 11th International Conference on Clouds and Precipitation, Miontreal, Canada. 


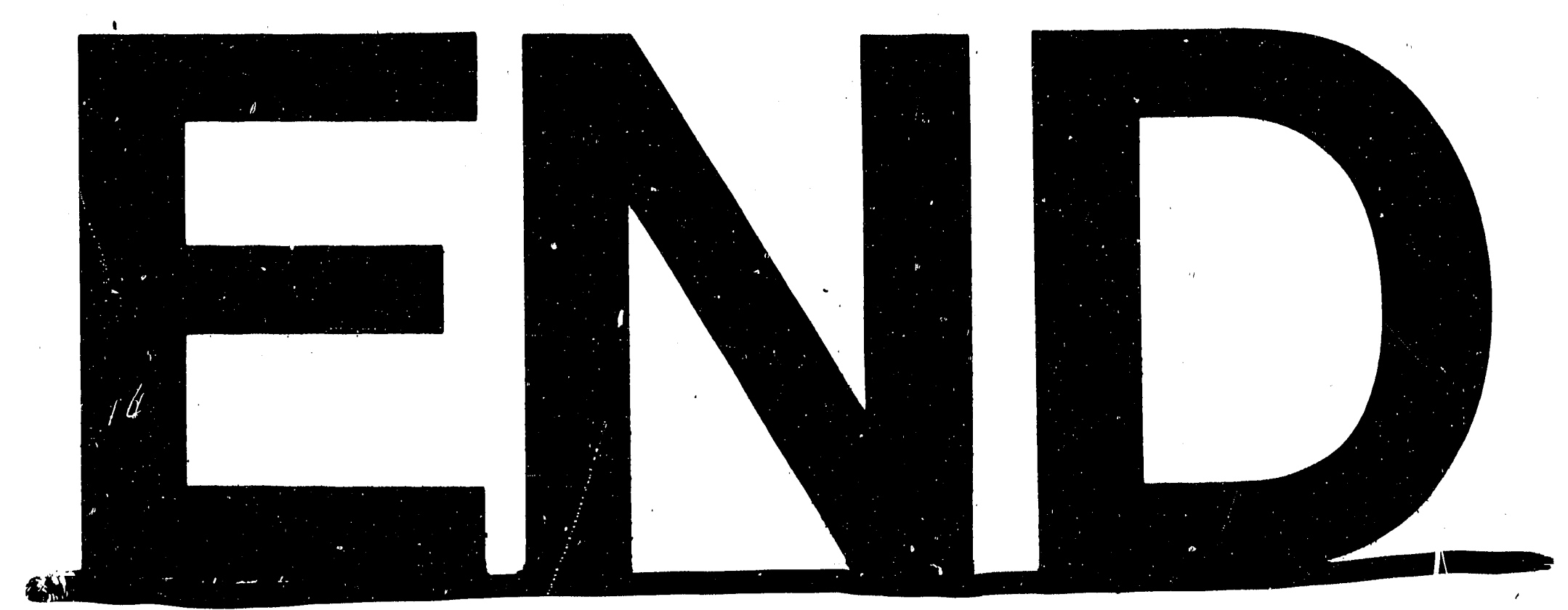

s.
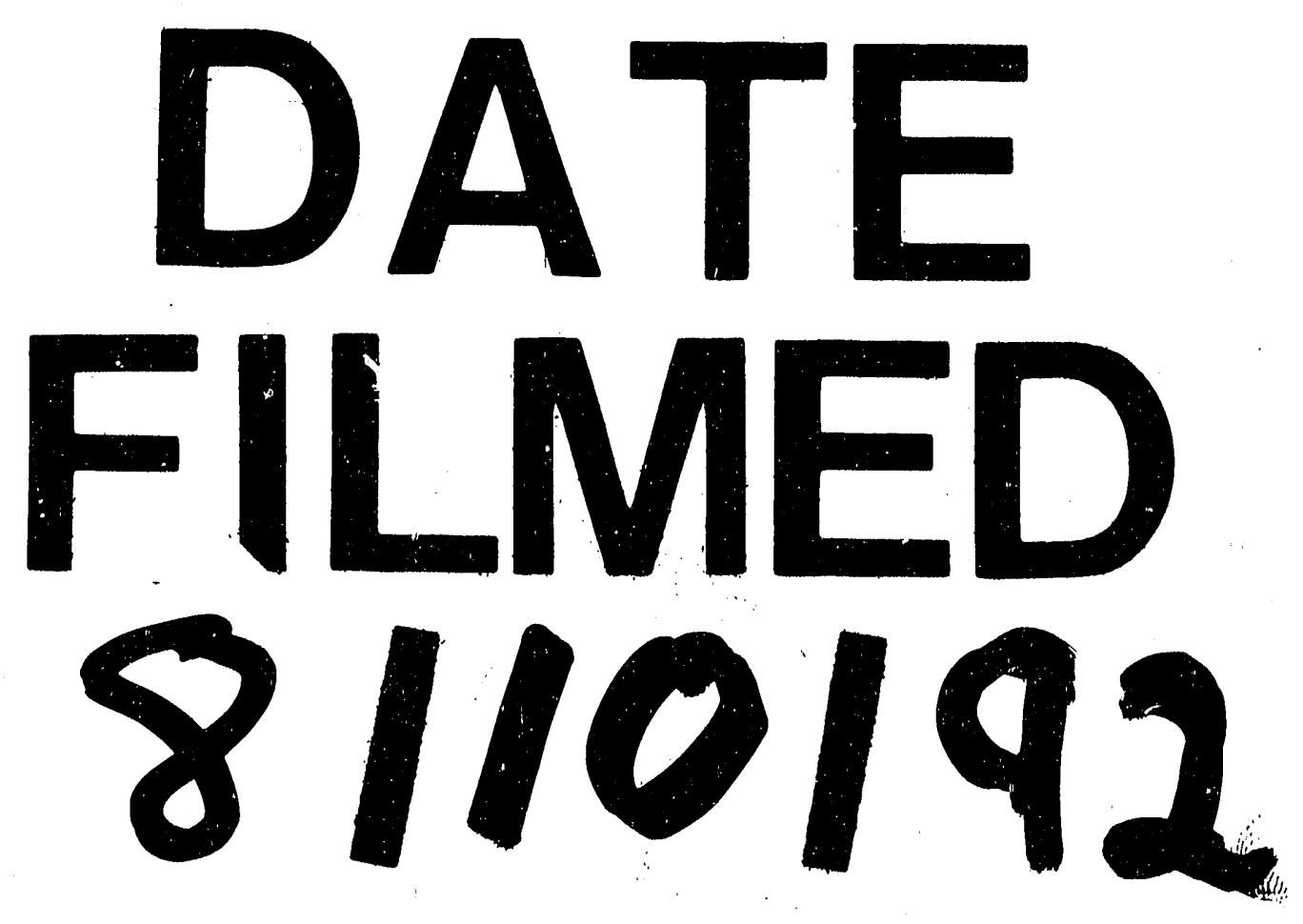

$=$ 
